\title{
On the trispectrum as a gaussian test for cosmology
}

\author{
Licia Verde \\ Depart. of Astrophysical sciences, Peyton Hall, Princeton University, Ivy lane, Princeton, NJ \\ 08544 1001, USA \\ Institute for Astronomy, University of Edinburgh, Blackford Hill, Edinburgh EH9 $3 H J$, UK \\ Iverde@astro.princeton.edu \\ Alan F. Heavens \\ Institute for Astronomy, University of Edinburgh, Blackford Hill, Edinburgh EH9 3HJ, UK \\ afh@roe.ac.uk
}

\begin{abstract}
In the standard model for structure formation, bound objects originate from the gravitational collapse of small perturbations arising from quantum fluctuations with random phases. In other scenarios, based on defects, structures are seeded by localized energy density. In principle, it is possible to differentiate between these models on the basis of their statistical properties; only in the former case is the initial density field an almost-perfect random gaussian field. In this paper, we investigate the use of the trispectrum of the galaxy density field, which is the connected four-point function in Fourier space, as a discriminant between gaussian and non-gaussian models. It has the advantage of having only weak non-linear growth. We define a related statistic $\tau$ which, as a test of the gaussian hypothesis, is independent of cosmology, the power spectrum and biasing, in real space, and which is, in principle, a measure of the departure from gaussian statistics. For galaxy redshift surveys, the statistic depends on cosmology and bias only through the potentially observable parameter $\beta$. We compute the expected errors on the estimate of $\tau$, and demonstrate with numerical simulations that it can be a useful discriminant of models, with the important proviso that any bias is linear on large scales. Whether it is the most effective method is uncertain and depends on the nature of the departure from gaussianity.
\end{abstract}

Subject headings: Cosmology: theory, large-scale structure of universe. Methods: analytical

\section{Introduction}

Models of primordial fluctuations that generated the large-scale cosmological structures can be divided into two broad classes: gaussian and non-gaussian. The simplest versions of inflationary 
cosmology predict almost perfectly gaussian initial fluctuations, but such fluctuations can arise under more general conditions from the central limit theorem. The popularity of gaussian models is also due to their mathematical simplicity and the possibility they present for analytical calculations. On the other hand, density fields generated by topological defects (Vilenkin 1985; Vachaspati 1986; Hill et al. 1989; Turok 1989; Albrecht \& Stebbins 1992) have non-gaussian initial conditions; the same can be said about fields generated by some versions of inflation (Allen et al. 1987; Kofman \& Pogosyan 1988; Salopek et al. 1989). Convincing evidence against gaussian initial conditions (GIC) would rule out many scenarios, and/or point us towards a physical theory for the origin of primordial fluctuations. Thus a test of the gaussian nature of the initial conditions is of great interest for cosmology.

Microwave background anisotropies probe cosmic fluctuations at a time when their statistical distribution should be close to its primeval form. To date, due to the limited signal-to-noise ratio of existing data, no conclusive evidence about the gaussianity of the initial conditions has been reached (e.g., Heavens (1998); Ferreira et al. (1998); Hinshaw et al. (1994); Falk et al. (1993); Luo \& Schramm (1993); Gangui et al. (1994); Luo (1994); Smoot et al. (1994); Kogut et al. (1996); Banday et al. (1999); Tegmark \& Bromley (1999)). An alternative is to analyze the present day density field of the galaxy distribution. This approach is complicated by the fact that the density field we observe today has already undergone non-linear gravitational evolution, and the observed non-gaussian nature of the galaxy distribution on small scales may be entirely the result of nonlinear gravitational clustering combined with biasing effects. This non-linear growth makes any intrinsic non-gaussian signal more difficult to detect, and leads one to the conclusion that generally study of the microwave background is likely to be more profitable in detecting non-gaussian features (Verde et al. 2000c). However, one cannot exclude the possibility that non-gaussian features arise on physical scales which are difficult to probe with the microwave background, or non-GIC might produce a gaussian Sachs-Wolfe effect (e.g., Scherrer \& Shaffer (1995)), so large-scale structure studies may still have a role to play in the test of the GIC hypothesis. An alternative method is to study number densities of rare/high-redshift objects (e.g., Chiu, Ostriker \& Strauss (1998); Robinson, Gawiser \& Silk (2000); Willick (2000); Matarrese et al. (2000); Verde et al. (2000a,b)).

There have been numerous studies to calculate the departures from gaussianity induced by gravity, and to set up a test of the gaussian nature of the initial conditions. There are essentially three different approaches: a) using N-body simulations, starting from GIC and several non-GIC, compare the resulting clustering properties of the evolved fields (e.g., Moscardini et al. (1991), Matarrese et al. (1991), Weinberg \& Cole (1992)) b) investigate the topological properties of fields generated from gaussian and non-gaussian conditions and set up a comparison (e.g., Moscardini et al. (1990); Coles et al. (1993); Vogeley et al. (1994); Avelino (1997)) c) measure the moments of the density or the velocity fields and compare them with those predicted from a gaussian distribution or different non-gaussian models (e.g., Fry (1984); Fry \& Scherrer (1994); Scherrer (1992); Catelan \& Moscardini (1994); Catelan \& Sherrer (1995), Gaztanaga \& Fossalba (1998a,b), Kim \& Strauss (1998); Bernardeau (1994)). 
In this paper, we take the last approach, but work in Fourier space. We concentrate on the 4-point function, for the following reasons. Under rather general conditions, the two-point function carries no model-independent information about the gaussian nature of the field (Fan \& Bardeen 1994). The bispectrum (3-point function) is more promising, as it is zero for a gaussian field, but generally non-zero. However, it grows at second-order in perturbation theory, and this signal will place limits on the accuracy with which one can identify a primordial component (Verde et al. 2000c). The trispectrum (4-point function) has the advantage that it grows linearly, with contributions from non-linear growth appearing only weakly in perturbation theory. This will be quantified in Sec. 2. One might hope, therefore, that the linear growth extends to relatively small scales. In addition, the possibility is open that non-gaussian models have no bispectrum, or even negative initial bispectrum (e.g., Moscardini et al. (1991)).

One can also probe the 4-point function in real space (e.g., Luo \& Schramm (1993); Lokas et al. (1995); Chodorowski \& Bouchet (1996)), or look at a subset of configurations of the four-point function, through power correlations (Feldman et al. 1994; Stirling \& Peacock 1996). An alternative way to approach mildly non-gaussian fields is based on the Edgeworth expansion (Amendola 1996).

The main difficulty with these approaches appears if the smoothed galaxy distribution is related to the underlying mass distribution by a non-linear transformation. In this circumstance, it is probably very difficult to distinguish non-GIC from a non-linear bias, so one has to assume that, at least on large scales, the bias is linear and deterministic (cf., Taruya et al. (2000)). In any eventuality, there is a realistic possibility that current large galaxy surveys such as the AngloAustralian 2-degree Field (hereafter 2dF; Colless (1996)) and the Sloan Digital Sky Survey (York et al. 2000) will place some constraints on any initial departures from gaussian behavior.

This paper is organized as follows: In section 2 we review the relevant statistical properties of the density field through its $n$-point functions, and introduce the $\tau$ statistic as a non-gaussian discriminant. Section 3 contains the practical implementation of the method on numerical simulations. Redshift-space distortions are considered in Section 4 and conclusions are presented in Section 5 .

\section{Statistics of the density field in the linear and weakly non-linear regime}

The statistical properties of the fractional overdensity field $\delta(\mathbf{x})=[\rho(\mathbf{x})-\bar{\rho}] / \bar{\rho}$, can be characterized by the $n$-point correlation functions or, in Fourier space, by the $n$-point spectra. If the fluctuation field is gaussian, the connected part of the $n$-point function vanishes for $n \geq 3$ (e.g., Bertschinger (1992)). Thus the two-point function, or alternatively the power spectrum, completely specifies a gaussian distribution.

To linear order in perturbation theory an initially gaussian distribution remains gaussian; in particular the the connected $n$-point functions and $n$-point spectra of an initially gaussian distribution are zero as long as linear perturbation theory holds. On the other hand, if the initial 
conditions are non-gaussian, the $n$-point spectra in the linear regime are the primordial ones, scaled by the $n^{\text {th }}$-power of the linear growth factor. Therefore it would be possible to detect primordial non-gaussianity with a measurement of non-zero connected linear $n$-spectra for $n \geq 3$.

We concentrate here on the four-point function in Fourier space (the trispectrum), because, as we demonstrate below, second-order contributions vanish for gaussian primordial fluctuations, so we hope to be able to use linear theory to smaller scales. This is tested numerically. Moreover this quantity can be made independent of the cosmological model, the degree of linear bias, and the power spectrum at least on large scales as we will show below.

The data which we will work with are products of four Fourier coefficients (in practice we use the real part of this):

$$
D_{\alpha}=\delta_{\mathbf{k}_{1}} \delta_{\mathbf{k}_{2}} \delta_{\mathbf{k}_{3}} \delta_{\mathbf{k}_{4}} .
$$

The mean value of this is zero (by homogeneity), unless the four wavevectors form a quadrilateral. $\alpha$ labels the set of 4 wavevectors.

Note that the trispectrum itself is strictly the Fourier counterpart of the connected part of the four-point correlation function only. For a zero-mean field with non-zero connected (subscript $c$ ) four-point function,

$$
\begin{aligned}
\left\langle\delta_{\mathbf{k}_{1}} \delta_{\mathbf{k}_{2}} \delta_{\mathbf{k}_{3}} \delta_{\mathbf{k}_{4}}\right\rangle & = \\
\left\langle\delta_{\mathbf{k}_{1}} \delta_{\mathbf{k}_{2}}\right\rangle\left\langle\delta_{\mathbf{k}_{3}} \delta_{\mathbf{k}_{4}}\right\rangle & +(2 \text { perms. })+\left\langle\delta_{\mathbf{k}_{1}} \delta_{\mathbf{k}_{2}} \delta_{\mathbf{k}_{3}} \delta_{\mathbf{k}_{4}}\right\rangle_{c}
\end{aligned}
$$

where

$$
\begin{aligned}
\left\langle\delta_{\mathbf{k}_{i}} \delta_{\mathbf{k}_{j}}\right\rangle & =(2 \pi)^{3} P\left(k_{i}\right) \delta^{D}\left(\mathbf{k}_{i}+\mathbf{k}_{j}\right) \\
\left\langle\delta_{\mathbf{k}_{1}} \delta_{\mathbf{k}_{2}} \delta_{\mathbf{k}_{3}} \delta_{\mathbf{k}_{4}}\right\rangle_{c} & =(2 \pi)^{3} T\left(\mathbf{k}_{i}\right) \delta^{D}\left(\mathbf{k}_{1}+\mathbf{k}_{2}+\mathbf{k}_{3}+\mathbf{k}_{4}\right) .
\end{aligned}
$$

Here $P(k)$ is the power spectrum, $T$ is the trispectrum, $\delta^{D}(\mathbf{k})$ is the three-dimensional Dirac delta function, the angle brackets indicate the ensemble average and $\left(\mathbf{k}_{i}\right)$ is a shorthand for $\left(\mathbf{k}_{1}, \mathbf{k}_{2}, \mathbf{k}_{3}, \mathbf{k}_{4}\right)$.

Note that $\left\langle\delta_{\mathbf{k}_{1}} \delta_{\mathbf{k}_{2}} \delta_{\mathbf{k}_{3}} \delta_{\mathbf{k}_{4}}\right\rangle$ has a specific volume dependence when one works with a discrete rather than continuous Fourier transform. The gaussian part involves products of two Dirac delta functions, whereas the connected part has only one. For a volume-limited survey of volume $V$, the discrete Fourier transform changes these delta functions to $\delta^{D} \longrightarrow V /(2 \pi)^{3}$ multiplied by a Kronecker delta, so any comparison between the size of non-gaussian and gaussian parts of $D_{\alpha}$ is volume-dependent. Any statement about the relative importance of the terms must therefore be made with some caution. We have to stress here that this is a general feature of $\left\langle\delta_{\mathbf{k}_{1}} \ldots \delta_{\mathbf{k}_{n}}\right\rangle$ that are evaluated in a finite volume: the relative importance of the connected and non-connected parts is volume dependent.

In order to demonstrate the lack of contribution to $\left\langle D_{\alpha}\right\rangle$ from second-order perturbation theory, we expand the density field to second order as:

$$
\delta(\mathbf{x})=\delta^{(1)}(\mathbf{x})+\delta^{(2)}(\mathbf{x})
$$


where $\delta^{(1)}$ is the linearly-evolved overdensity. $\delta^{(2)}$ is $O\left(\delta^{(1) 2}\right)$ and, if initial conditions are gaussian, represents departures from gaussian behavior due to gravitational evolution (e.g., Goroff et al. (1986), Bouchet et al. (1992)). Strictly speaking the galaxy distribution is not a mildly non-linear field, but a highly non-linear field filtered on some smoothing scale. The operations of smoothing and evolution do not commute, but experiments (e.g., Matarrese et al. (1997), hereafter MVH97) show that perturbation theory works well if the smoothed field is not too non-linear.

To second order in perturbation theory the Fourier counterpart of the connected four-point correlation function can be obtained by applying to eq. (2) the substitution

$$
\delta_{\mathbf{k}}=\delta_{\mathbf{k}}^{(1)}+\epsilon \delta_{\mathbf{k}}^{(2)}+\epsilon^{2} \delta_{\mathbf{k}}^{(3)}+\ldots
$$

and retaining only terms $\propto \epsilon$. Here $\epsilon$ is simply a bookkeeping parameter that can then be set to unity and the expression for $\delta_{\mathbf{k}}^{(2)}$ can be found e.g., in Fry (1984) and Catelan et al. (1995). Hence

$$
\begin{aligned}
\left\langle\delta_{\mathbf{k}_{1}} \delta_{\mathbf{k}_{2}} \delta_{\mathbf{k}_{3}} \delta_{\mathbf{k}_{4}}\right\rangle= & \left\langle\delta_{\mathbf{k}_{1}}^{(1)} \delta_{\mathbf{k}_{2}}^{(1)} \delta_{\mathbf{k}_{3}}^{(1)} \delta_{\mathbf{k}_{4}}^{(1)}\right\rangle+ \\
& \left\langle\delta_{\mathbf{k}_{1}}^{(1)} \delta_{\mathbf{k}_{2}}^{(1)} \delta_{\mathbf{k}_{3}}^{(1)} \delta_{\mathbf{k}_{4}}^{(2)}\right\rangle+c y c .(4 \text { terms }) .
\end{aligned}
$$

There are 4 cyclical terms involving the second order $\delta_{\mathbf{k}}^{(2)}$ whose expression involves products of two linear coefficients, so for an initially gaussian field, the second-order contributions to the trispectrum are products of five coefficients and hence vanish. The first non-vanishing contribution to the connected trispectrum due to gravitational instability is $\propto \epsilon^{2}$ as is the next (third order) term in (5). In general, the trispectrum will depend on all orders of correlations present initially, but in the linear regime, the only contribution is from the initial trispectrum $\left(T_{\text {initial }}\right)$. Specifically, if $\mathcal{D}(t)$ is the growth factor:

$$
T=T_{\text {initial }} \mathcal{D}(t)^{4} \text {. }
$$

For GIC, the trispectrum is zero, but $\left\langle D_{\alpha}\right\rangle_{G I C}$ has a disconnected part

$$
\left\langle D_{\alpha}\right\rangle_{G I C}=(2 \pi)^{6} P\left(k_{1}\right) P\left(k_{3}\right) \delta^{D}\left(\mathbf{k}_{1}+\mathbf{k}_{2}\right) \delta^{D}\left(\mathbf{k}_{3}+\mathbf{k}_{4}\right)+c y c .
$$

If primordial fluctuations are non-gaussian, then $\left\langle D_{\alpha}\right\rangle \neq\left\langle D_{\alpha}\right\rangle_{G I C}$. For $k$-vector configurations where all $|\mathbf{k}|$ are different, $\left\langle D_{\alpha}\right\rangle_{G I C}$ vanishes, leaving $\left\langle D_{\alpha}\right\rangle=(2 \pi)^{3} T\left(\mathbf{k}_{1}, \mathbf{k}_{2}, \mathbf{k}_{3}, \mathbf{k}_{4}\right) \delta^{D}$. We can thus characterize the departure from gaussian behavior by

$$
\begin{aligned}
& \left\langle\delta_{\mathbf{k}_{1}} \delta_{\mathbf{k}_{2}} \delta_{\mathbf{k}_{3}} \delta_{\mathbf{k}_{4}}\right\rangle \\
= & (2 \pi)^{6} P\left(k_{1}\right) P\left(k_{2}\right) \delta^{D}\left(\mathbf{k}_{1}+\mathbf{k}_{3}\right) \delta^{D}\left(\mathbf{k}_{2}+\mathbf{k}_{4}\right)+c y c . \\
+ & \tau \sqrt{P\left(k_{1}\right) P\left(k_{2}\right) P\left(k_{3}\right) P\left(k_{4}\right)} \delta^{D}\left(\mathbf{k}_{1}+\mathbf{k}_{2}+\mathbf{k}_{3}+\mathbf{k}_{4}\right),
\end{aligned}
$$

where we have introduced the quantity

$$
\tau \equiv \frac{T\left(\mathbf{k}_{1}, \mathbf{k}_{2}, \mathbf{k}_{3}, \mathbf{k}_{4}\right)}{\sqrt{P\left(k_{1}\right) P\left(k_{2}\right) P\left(k_{3}\right) P\left(k_{4}\right)}} .
$$


$\tau$ is independent of the volume, the cosmological model, linear bias and redshift (or alternatively the power spectrum amplitude), but in general depends on the $\mathbf{k}_{i}$ (i.e. the scale and the configuration of the quadrilateral formed by the $4 k$-vectors). Here for simplicity we will use equilateral k-vector configurations; with this choice isotropy demands that $\tau$ depends only on $|\mathbf{k}|$, for a given shape. We can then use a likelihood method to estimate $\tau(k)$, and deduce whether it is consistent with the gaussian result $(\tau=0)$.

\subsection{Likelihood}

Our treatment here follows that of MVH97. For simplicity, we would like to be able to adopt a gaussian likelihood (italics will refer to the form of the likelihood function, not the initial statistics), which will be the a posteriori probability distribution for $\tau$ if we assume a uniform prior:

$$
\begin{aligned}
\mathcal{L}(\tau)= & \frac{1}{(2 \pi)^{\frac{M}{2}}(\operatorname{det} C)^{\frac{1}{2}}} \times \\
& \exp \left[-\frac{1}{2} \sum_{\alpha \beta}\left(D_{\alpha}-\mu_{\alpha}\right) C_{\alpha \beta}^{-1}\left(D_{\beta}-\mu_{\beta}\right)\right] .
\end{aligned}
$$

Here $M$ is the number of data, which have means $\mu_{\alpha}(\tau) \equiv\left\langle D_{\alpha}\right\rangle$, and that can be evaluated using equation 9. The covariance matrix is $C_{\alpha \beta} \equiv\left\langle\left(D_{\alpha}-\mu_{\alpha}\right)\left(D_{\beta}-\mu_{\beta}\right)\right\rangle$. If $\tau=0$ the likelihood analysis is effectively a $\chi^{2}$ analysis.

In the general (i.e. non-gaussian) case we cannot justify the applicability of the gaussian likelihood mathematically from the central limit theorem, but numerical experiments (section 3) support its use when many modes are used.

The covariance matrix involves the eight-point correlation function, computable from Wick's theorem and, for continuous and discrete fields by the methods of MVH97:

$$
\begin{array}{cc}
\left\langle\delta_{1} \ldots . \delta_{8}\right\rangle_{\text {total }}= & \\
\left\langle\delta_{1} \delta_{2}\right\rangle\left\langle\delta_{3} \delta_{4}\right\rangle\left\langle\delta_{5} \delta_{6}\right\rangle\left\langle\delta_{7} \delta_{8}\right\rangle+\ldots & 105 \text { terms } \\
+\left\langle\delta_{1} \delta_{2}\right\rangle\left\langle\delta_{3} \delta_{4} \delta_{5}\right\rangle\left\langle\delta_{6} \delta_{7} \delta_{8}\right\rangle+\ldots & 280 \text { terms } \\
+\left\langle\delta_{1} \delta_{2}\right\rangle\left\langle\delta_{3} \delta_{4} \delta_{5} \delta_{6}\right\rangle\left\langle\delta_{7} \delta_{8}\right\rangle+\ldots & 210 \text { terms } \\
+\left\langle\delta_{1} \delta_{2} \delta_{3} \delta_{4}\right\rangle\left\langle\delta_{5} \delta_{6} \delta_{7} \delta_{8}\right\rangle+\ldots & 35 \text { terms } \\
+\left\langle\delta_{1} \delta_{2}\right\rangle\left\langle\delta_{3} \delta_{4} \delta_{5} \delta_{6} \delta_{7} \delta_{8}\right\rangle+\ldots & 28 \text { terms } \\
+\left\langle\delta_{1} \delta_{2} \delta_{3}\right\rangle\left\langle\delta_{4} \delta_{5} \delta_{6} \delta_{7} \delta_{8}\right\rangle+\ldots & 56 \text { terms } \\
& +\left\langle\delta_{1} \ldots \delta_{8}\right\rangle .
\end{array}
$$

These expressions apply both to continuous fields and discrete point processes, and the averages on 
the r.h.s. are all connected parts only. Under the hypothesis of GIC, as long as linear theory holds ${ }^{1}$, all terms involving the connected $m$-point correlation with $m$ greater or equal than two are nonzero only in the presence of shot noise. Moreover for equal-side configurations the terms involving $\left\langle\delta_{l} \delta_{m} \delta_{n}\right\rangle$ are also zero, since one cannot make a triangle from any 3 of the wavevectors. For clarity we neglect shot noise in the main text, but the calculations have been performed including it.

For GIC, the only non-zero terms come from the two-point function, i.e. the power spectrum. We can test the gaussian hypothesis by including only these gaussian terms in the covariance matrix, reducing the process to $\chi^{2}$ analysis. This should also be a good approximation for mildly non-gaussian fields (cf. Heavens (1998) for the microwave background). With this covariance matrix, a measurement of $\tau$ which is significantly non-zero would only rule out gaussian statistics, but would not necessarily recover the correct value of $\tau$, which would require use of the correct non-gaussian covariance matrix.

\subsection{A priori estimation of the error on $\tau$}

We can readily calculate the expected variance of our estimator for $\tau$. The calculation follows MVH97 so we only sketch the calculation here. Ignoring shot noise, for square configurations we have

$$
\mu_{\alpha}=\left\langle D_{\alpha}\right\rangle=(2 \pi)^{6} P^{2}\left(\delta^{D}\right)^{2}+(2 \pi)^{3} \tau P^{2} \delta^{D}
$$

and the variance is:

$$
\sigma_{\alpha}^{2}=\left\langle D_{\alpha}^{2}\right\rangle=4 P^{4}\left[(2 \pi)^{3} \delta^{D}\right]^{4}
$$

since only 4 terms are non-zero out of the 105 . Hence

$$
\sigma_{\tau}^{-2}=-\left\langle\frac{\partial^{2} \ln \mathcal{L}}{\partial \tau^{2}}\right\rangle_{\tau=0}=\sum_{\alpha} \frac{\mu_{\alpha}(\tau=0) P^{2}}{\sigma_{\alpha}^{2}} .
$$

The number of uncorrelated squares in a thin shell in $k$-space of width $\delta(\ln k)$ is $\pi k^{3} g \delta(\ln k)$ where $g=V /(2 \pi)^{3}$ is the density of states. Considering contributions from all the shells to the continuum limit we obtain

$$
\sigma_{\tau}^{-2}=\frac{1}{2^{3} \pi^{2}} \frac{1}{4 V} \int_{k_{\min }}^{k_{\max }} k^{3} d(\ln k) .
$$

$k_{\max }$ is set by the breakdown of second-order perturbation theory and $k_{\min }$ by the size of the sample. Therefore the error on $\tau$ scales as $\sqrt{V}$ and the maximum signal-to-noise is obtained by splitting the volume into subunits. This procedure to reduce the error when dealing with higherorder spectral statistics is widely used in other fields such as signal processing ${ }^{2}$ and fluid mechanics

\footnotetext{
${ }^{1}$ As shown above, second order contributions vanish for gaussian primordial fluctuations, therefore linear theory breaks down when third-order contributions become important.

${ }^{2}$ Where it is referred to as segment averaging.
} 
see e.g., Brillinger (1975), Brillinger \& Rosenblatt (1967), Lii et al. (1976) and has been already applied in cosmology by Matarrese et al. (1997) and Verde et al. (1998). This appears to give something for nothing, but of course this is not true. The resolution of the paradox is given in section 2.3.

The minimum size of the samples may be set by the breakdown of perturbation theory, or possibly by aliasing difficulties if there is significant power on scales larger than the box.

Note that the analysis in this section, and therefore the scaling (16), holds only as long as the field is close to gaussian so that non-gaussian elements of the covariance matrix can be neglected. As we said before, if the field is close to gaussian we are justified in adopting a gaussian likelihood, however if the distribution appears to be highly non-gaussian this error calculation and volume dependence is no longer valid. Since effectively $P(k)$ is estimated from the data themselves, in principle one should propagate any error on $P(k)$ through into the error on $\tau$. However, since the cosmic variance for the trispectrum is much larger than that on the power spectrum, this effect should be negligible. We confirmed this with numerical experiments.

The square configuration we considered above is not the only possible one. We could also consider other configurations in which all the $k$-vectors in the quadruplet have the same modulus. These configurations can be parametrized by the angle $\theta$ between the first two vectors and $90^{\circ} \geq$ $\theta \geq 0^{\circ}$ : in the square case $\theta=90^{\circ}$, in the 'degenerate' case $\theta=0^{\circ}$. The above error analysis holds for all $\theta>0^{\circ}$. For the 'degenerate' case of $\theta=0^{\circ}$, there are modifications to the number of cyclic terms contributing to the means and covariance matrix, and also to the number of independent quadrilaterals. In this case, we have $\mu_{\alpha}=2 P^{2}\left[(2 \pi)^{3} \delta^{D}\right]^{2}+(2 \pi)^{3} \tau P^{2} \delta^{D}, \sigma_{\alpha}^{2}=24 P^{4}\left[(2 \pi)^{3} \delta^{D}\right]^{4}$, and the number of uncorrelated quadruplets is $2 \pi k^{3} g \delta(\ln k)$. These reduce the error by a factor $\sqrt{2 \times 2^{4} / 24}=1.15$, if $k_{\max }$ remains the same.

\subsection{Volume-splitting procedure}

Volume-splitting appears to improve the signal-to-noise. We show here in outline that it gives no more information than relaxing the fixed quadrilateral shape and including more modes.

Consider the $n$ point spectrum and imagine that we wish to compute the expected error on the amplitude $A$ : $\left\langle\delta_{k_{1}} \ldots \delta_{k_{n}}\right\rangle=A S \delta^{D}$ where $\delta^{D} \propto V$ and $S$ is some fixed function. The leading term in the covariance, neglecting shot noise, will be $\sim P^{n}\left(\delta^{D}\right)^{n}$. As already seen before the density of states is $g \propto V$. If we fix the configuration of the $k$-vectors, then the expected error on $A$, for a thin shell in $k$-space, will be given by:

$$
\sigma_{A}^{-2} \propto \frac{g S^{2}\left(\delta^{D}\right)^{2}}{P^{n}\left(\delta^{D}\right)^{n}}
$$

which implies the curious result that $\sigma_{A} \propto V^{(n-3) / 2}$. However, if we allow the shape of the $k$-vector 
configuration to change we obtain:

$$
\sigma_{A}^{-2} \propto \frac{g^{(n-1)} S^{2}\left(\delta^{D}\right)^{2}}{P^{n}\left(\delta^{D}\right)^{n}}
$$

where the exponent $n-1$ of the density of states comes from the presence of the Dirac delta function, i.e. the fact that the polygon has to close and therefore has $n-1$ degrees of freedom. In this case we obtain the (more intuitive result) that: $\sigma_{A} \propto 1 / \sqrt{V}$ when all shapes are allowed for.

In the case of the power spectrum (as in the example of Press et al. (1992)) the two possibilities coincide $(n=1)$, for this reason splitting the volume does not improve the errors. Note also that in the bispectrum case $(n=3)$ of Matarrese et al. (1997) and Verde et al. (1998), the error is independent of the size of the sample because the triangle shape is fixed. For the trispectrum $(n=4) \sigma_{A} \propto \sqrt{V}$. Here, as in all cases, one has the choice between considering all shapes, or keeping the shape fixed and subdividing.

\section{N-body tests}

In this section, we perform tests with real-space results from N-body simulations. We analyze square configurations, and ensure that no wavevector occupies more than one square, so the covariance matrix is approximately diagonal (cf. MVH97 for the bispectrum). The simulation is a $128^{3}$ particle, $100 h^{-1}$ Mpc side box, CDM-like simulation from the Hydra consortium (Couchman et al. 1995), with parameters $\Omega=1.0, \Lambda=0.0, \sigma_{8}=0.64, \Gamma=0.25$, and GIC. Shot noise is completely negligible in all scales of interest $(k<1$; here and hereafter $k$ is in units of $\mathrm{h} / \mathrm{Mpc})$.

The breakdown of linear theory for the trispectrum is not known in advance, but from MVH97 one can expect that the leading-order corrections for the trispectrum (i.e. contributions from the third order in perturbation theory) should be small at least up to $k=0.55 \mathrm{~h} \mathrm{Mpc}^{-1}$. A likelihood computation (cf., section 2.1) shows that for this square configuration, linear theory for the 4-point function should be valid up to $k_{\max }=0.67$, which sets the upper limit for our further analysis, giving 1250 squares in total. This limit is also apparent in Fig. 1, which plots ${ }^{3}\left\langle D_{\alpha}\right\rangle$. For this wavenumber limit, $\chi^{2}$ is shown as a function of $\tau$ in Fig. 2: the minimum reduced $\chi^{2}$ is 0.8 and $\tau=(-1.5 \pm 5.5) \times 10^{4}$ is consistent with zero. The a priori estimation for the error of Eq.(16) yields $\sigma_{\tau}=5.6 \times 10^{4}$. Note that the estimate of $\tau$ is independent of linear biasing because of the presence of the same number of $\delta$ factors in the numerator and denominator of Eq. 10.

We have also analyzed another N-body simulation with very non-GIC. The initial conditions

\footnotetext{
${ }^{3}$ The notation here follows MVJ97: $D_{\alpha}$ is a single measurement of a statistical quantity i.e. the trispectrum for a given (e.g., square) $k$ configuration on a given scale (e.g., $k=0.5) .\left\langle D_{\alpha}\right\rangle$ is the ensemble average of this quantity. Since we assume that the volume under consideration is a "fair sample", then the average of all $D_{\alpha}$ in the simulation on the same scale and with the same $k$ configuration is an estimate of $\left\langle D_{\alpha}\right\rangle$.
} 
were set by applying the following mapping to a gaussian field:

$$
\delta \longrightarrow \delta^{2}-\left\langle\delta^{2}\right\rangle
$$

(the so-called $\chi^{2}$ model for initial conditions). The linear power spectrum is a power law with spectral index $n=-1$. The simulation we used has $80^{3}$ particles, in a $200 h^{-1} \mathrm{Mpc}$ side box, with $\sigma_{8}=0.5$. In this simulation the shot noise is not negligible, but it can be included with the methods of MVH97. This family of initial conditions could arise from evolution through inflation of an isocurvature CDM model for structure formation and is motivated by the fact that it accommodates galaxy formation at high redshift (e.g., Peebles (1999a,b)). Despite the low normalization, in this case we find that for all $k$-space shells with $k<0.67$ the minimum $\chi^{2}$ value for $\tau$ is nonzero at high significance: considering all $k$-vectors in the range $0.15<k<0.67$ for equilateral configurations and in the range $0.15<k<0.75$ for degenerate configurations, we obtain $\tau_{e q}=(9.2 \pm 1.6) \times 10^{5}$ and $\tau_{d e g}=(6.4 \pm 1.3) \times 10^{5}$ respectively. Thus we can reject the GIC hypothesis with confidence, but note that for this highly non-gaussian model the covariance matrix is not accurate, so we can not rely on the actual measurement of $\tau$ being reliable.

Incidentally, for this simulation, the bispectrum also differs in the mildly non-linear regime from the one that would have originated by gravitational instability from GIC. However, at the bispectrum level, there is degeneracy between biasing and initial non gaussianity: in practice it would not be possible to assess if there is substantial bias/anti-bias or if the initial conditions were truly non-gaussian. As we have mentioned, the trispectrum method is bias-independent, at least as long as the bias is not strongly non-linear.

\subsection{Subdividing the Volume}

We have tested the subdivision procedure with static simulations of a non-gaussian density field derived from a gaussian $\delta(x)$

$$
\delta(x) \longrightarrow \delta(x)+\epsilon \delta^{2}(x)
$$

where $\epsilon$ is some parameter. For simplicity, we take a white noise power spectrum between $k_{\min }$ and $k_{\max }$. Details of the resulting 2-point and 4-point functions are given in the Appendix, but they are summarized as

$$
P(k)=P_{g}(k)+2 \epsilon^{2} P_{g}(k)^{2} V_{k} /(2 \pi)^{3}
$$

where $P_{g}(k)$ is the power spectrum of the underlying gaussian $\delta$ field, and $V_{k}$ is a $k$-space volume defined in the appendix. The 4-point function is

$$
\left\langle\delta_{\mathbf{k}_{1}} \delta_{\mathbf{k}_{2}} \delta_{\mathbf{k}_{3}} \delta_{\mathbf{k}_{4}}\right\rangle_{\text {conn. }} \sim\left(48 \epsilon^{2} P_{g}^{3}+\epsilon^{4} P_{g}^{4} V_{k} /(2 \pi)^{3}\right) V .
$$

We choose $P_{g}=2(2 \pi)^{3}$ in the range $0.1<k<0.837$ and zero elsewhere, and $\epsilon=0.4$. In this case, the non-gaussian terms in the covariance matrix are small in comparison with the gaussian 
one and we ignore them. We split the volumes into cubes of side 20 and $24 h^{-1} \mathrm{Mpc}$, and make repeated simulations to reduce errors. In all, we analyzed 460 of the smaller boxes and 266 of the larger boxes, so the total volume is the same in the two cases. The results are shown in Fig. 3. The expected value of $\tau$ is 1040, and is recovered within the errors. Note also that the errors (160 and 250 respectively) scale as expected $\propto N$ where $N$ is the number of boxes, and the signal-to-noise is indeed higher for the ensemble of smaller boxes, as expected.

\section{Complications with a real survey}

In section 3 we showed that the trispectrum is a useful discriminant between GIC and nonGIC in a very idealized case where the field is unbiased, and the positions of the particles are known in real space. Note however that galaxy catalogues have an average number of galaxies per unit volume that is about two orders of magnitude smaller than the one of the simulation used, and use the redshift as a third spatial coordinate. The resulting redshift-space map of the galaxy distribution is therefore distorted and shot noise is significant. This is not a trivial issue since we are pushing into the mildly non-linear regime, which is significantly affected by non-perturbative contributions.

In this section, we show how to deal with these effects.

\subsection{Redshift-space distortions}

It is a convenient approximation to split redshift-space distortions into two components (Kaiser 1987): a large scale distortion responsible of the squashing known also as the 'bull's eye' effect and a small-scale radial smearing responsible for 'Fingers-of-God'. The large-scale effect on individual Fourier components of the density fluctuation is well described by multiplication by the Kaiser factor $\left(1+\beta \mu^{2}\right)$, where $\mu$ is the cosine of the angle between the $k$-vector and the line of sight, and $\beta \equiv \Omega_{0}^{0.6} / b$ with $b$ the linear bias parameter.

The small-scale effect is hard to treat exactly and could potentially erase the signal we are trying to detect; in fact virialized motions on small scales produce a radial smearing and the associated Finger-of-God effect contaminates the wavelengths we are interested in. A successful model that fits the power spectrum in numerical simulation reasonably well (e.g., Hatton \& Cole (1998); Peacock \& Dodds (1994)) assumes that the small-scale velocity field is uncorrelated with density and has an exponential velocity distribution. Although not rigorously theoretically-motivated, it has been

shown in VHMM98 that this modeling of the small-scale velocity dispersion works well as an addition to perturbation theory. In the Fourier domain, and in the distant observer approximation, the exponential velocity dispersion gives a damping factor, that, combined with the boosting factor 
of the large-scale effect (Kaiser 1987) gives (Peacock \& Dodds 1994):

$$
\delta_{\mathbf{k}} \longrightarrow \delta_{\mathbf{k}} \frac{\left(1+\beta \mu^{2}\right)}{\sqrt{1+k^{2} \sigma^{2} \mu^{2} / 2}},
$$

where $\sigma$ is the pairwise velocity dispersion of galaxies. With this model for redshift-space distortions:

$$
\left\langle D_{\alpha}\right\rangle(k) \longrightarrow\left\langle D_{\alpha}\right\rangle(k, \mu)=\left\langle D_{\alpha}\right\rangle(k) \frac{\left(1+\beta \mu^{2}\right)^{4}}{\sqrt{\prod_{i=1, . ., 4}\left(1+k_{i}^{2} \sigma^{2} \mu_{i}^{2} / 2\right)}} .
$$

As in VHMM98 we allow $\sigma$ to be scale-dependent (to fit the power spectrum - there is some observational evidence for this; see Hamilton \& Tegmark (2000)) and we reject the $k$-vectors aligned too closely with the line-of-sight (see Scoccimarro et al. (1999) for an alternative model).

We have tested the model (24) for square configurations by performing a $\chi^{2}$ analysis for the parameter $\tau$ on the unbiased redshift-space catalogue created from the N-body simulation with GIC.

Following VHMM98 the covariance matrix is modified as follows. The power spectrum is first replaced by $P(\mathbf{k}) \equiv P(k)\left(1+\beta \mu^{2}\right)^{2}$ and the resulting covariance matrix is then divided by $\sqrt{\prod_{i=1}^{8}\left(1+k_{i}^{2} \sigma^{2} \mu_{i}^{2} / 2\right)}$, where the index $i$ runs over the $8 k$-vectors that form the two squares.

The knowledge of the real-space power spectrum is required in this model: in a realistic application (like for example Sloan and $2 \mathrm{dF}$ ) an accurate fit for the galaxy real-space power spectrum will be known. Thus for the present work we shall assume that the real-space power spectrum is known: only the velocity dispersion $\sigma$ needs to be determined, and this can be done by fitting the power spectrum. The limit of validity of the small-scale redshift-distortion model for the trispectrum can also in principle be determined, although in this case the limiting factor is the breakdown of perturbation theory. A likelihood analysis of the redshift-space Fourier modes can give $\sigma$ in a similar manner to VHMM98.

The result for the redshift-space analysis is shown in Fig. 4. Again the true value for $\tau$ is well recovered within the errors: $\tau=(-1.0 \pm 5.5) \times 10^{4}$ (reduced $\chi^{2}$ value is 0.95 ) for the square configuration and $\tau=(0.8 \pm 4.5) \times 10^{4}$, for the degenerate configuration in good agreement with the predicted error of $4.3 \times 10^{4}$ obtained as outlined in section 2.2 .

As a test of the velocity dispersion, we consider the quadrupole-to-monopole ratio of $\left\langle D_{\alpha}\right\rangle$, following its use with the power spectrum Hamilton (1992) and the bispectrum (VHMM98, Scoccimarro et al. (1998)). For the degenerate configuration all vectors have the same $\mu^{2}$, so the quadrupole-to-monopole ratio can be simply defined by $R_{T} \equiv\left\langle D_{\alpha}\right\rangle^{(2)} /\left\langle D_{\alpha}\right\rangle^{(0)}$, where the quadrupole and monopole moments of $T$ are

$$
\begin{aligned}
\left\langle D_{\alpha}\right\rangle^{(2)} & =\frac{5}{2} \int_{-1}^{1}\left\langle D_{\alpha}\right\rangle(k, \mu)\left(3 \mu^{2}-1\right) d \mu \\
\left\langle D_{\alpha}\right\rangle^{(0)} & =\frac{1}{2} \int_{-1}^{1}\left\langle D_{\alpha}\right\rangle(k, \mu) d \mu .
\end{aligned}
$$


In Fig. 5 we show how the model (24) reproduces the observed $R_{T}$ in the GIC simulation. We follow the same procedure as VHMM98, where $\sigma$ is allowed to be slightly scale-dependent, constrained to fit the power spectrum, and $k^{2} \mu^{2} \leq 0.3$. It would be possible to define a similar quantity also for non-degenerate configurations, but in this case $\left\langle D_{\alpha}\right\rangle$ in redshift space would depend on two different angles to the line-of-sight which leads to more complexity.

For the non-gaussian isocurvature field of Section 3.1, we find a $\tau$ which is inconsistent with zero: e.g., for degenerate configurations $\tau_{\text {deg }}=(6.4 \pm 1.3) \times 10^{5}$. For non-gaussian models, in general, the signal $\tau$ will be shape-dependent, (as for example in the particular case illustrated in section $5)$. This dependence on the $k$-vector configuration might therefore hold some extra information or allow stronger detection of a non-gaussian signal.

\subsection{Expected performance from PSCz and Sloan}

The PSCz survey (Saunders et al. 1998) is the largest nearly-all-sky survey, containing around 15000 galaxies. The space density of galaxies is not very high, so shot noise is important beyond about $40 h^{-1} \mathrm{Mpc}$ for $k=0.67$. Assuming the initial field is close to gaussian, we use the gaussian covariance matrix, but with shot noise included.

Before being able to perform the trispectrum analysis on this survey however still there are a number of unresolved issues. The most important are the practical effects of the rapidly-varying selection function and the radial nature of the redshift-space distortions. In fact the technique described in section 4.1 assumes that the spherical nature of the distortion can be neglected and the sky can be considered flat (the so-called 'distant-observer approximation', or 'plane-parallel approximation'). We must emphasize that this approximation does not hold for the $\mathrm{PSCz}$, and one really has to go beyond the plane-parallel approximation, by using spherical harmonics for example (e.g., Heavens \& Taylor (1995)).

In the case of the $2 \mathrm{dF}$ and Sloan surveys, due to the bigger volume available, the problem of the radial nature of the redshift-space distortions is much reduced, and the selection function issue can be tackled as illustrated in the appendix. The $1 \sigma$ error achievable on $\tau$ is very encouraging: $1 \times 10^{4}$ for Sloan, if we restrict the analysis to the linear regime $(k<0.3 h)$ and divide the survey conservatively into $100 h^{-1} \mathrm{Mpc}$ side cubes. This reduces to $4 \times 10^{3}$ if wavenumbers up to $k=0.7 \mathrm{~h}$ $\mathrm{Mpc}^{-1}$ are included. The error can be reduced even further by considering smaller boxes.

\subsubsection{Meaning of non zero value for $\tau$}

Most inflationary models predict deviations from gaussianity of the form described by equation (26)-applied to the potential rather than the density fluctuation field; this kind of non-gaussianity affects mainly the bispectrum (e.g., Verde et al. (2000c)). An order of magnitude calculation shows 
that for the non-gaussian models analyzed in Verde et al. (2000c), the minimum deviation from gaussianity that can be detected with the large-scale-structure bispectrum is at least a factor of a few smaller than the one detectable with the large-scale-structure trispectrum; in these cases an analysis of the cosmic microwave background bispectrum is thus more promising. However, as already mentioned, primordial non-gaussianity might arise on physical scales which are difficult to probe with the microwave background, the large-scale-structure bispectrum might deviate from the perturbation theory prediction due to the presence of bias or antibias, and non-gaussian models might have no primordial bispectrum, or even negative initial bispectrum. It is nevertheless clear that other plausible non-gaussian models such as the $\chi^{2}$ model described in section 3 can easily be ruled out with a trispectrum analysis of Sloan $/ 2 \mathrm{dF}$ data sets.

\section{Conclusions}

We have presented a method for using the trispectrum, the four-point function in Fourier space, to discriminate between GIC and non-GIC from linear large-scale structure data. The advantage of this method is that for mildly non-gaussian fields the analysis in real space is independent of the power spectrum normalization, linear biasing, and cosmology, and, as a spectral method, the covariance matrix is more simply computed, in contrast to $n$-point correlation functions. In redshift space, cosmology and bias enter only through the measurable quantity $\beta$, we show how to deal with redshift-space distortions (in the distant-observer approximation), we include in the calculations effects of shot noise following the method presented in MVH97 (although for clarity the shot noise contribution is ignored in the text and reported in the appendix) and it is straightforward to model the effects of varying selection function using the method of MVH97 (see the Appendix). The non-linear evolution of the trispectrum is expected to be rather weak in perturbation theory, so our expectation is that linear theory should provide a good description up to scales where the field becomes significantly non-linear. This is born out (and quantified) in simulations. Fig. (1) illustrates this point. The equilateral configurations bispectrum for the same simulation considered here, agrees with second-order perturbation theory up to $k \sim 0.55$ (MVH97). Fig (1) shows linear perturbation theory is adequate for square trispectrum configurations up to $k \sim 0.67$. We parameterize the departures from gaussian statistics by introducing the related quantity $\tau$, which is the 4-point correlation function in units of $\sqrt{P\left(k_{1}\right) P\left(k_{2}\right) P\left(k_{3}\right) P\left(k_{4}\right)}$. This quantity, in specific cases, can give us a meaningful measure of 'non-gaussianity'. We compute the gaussian variance of $\tau$, which allows a test of the gaussian hypothesis. The error on $\tau$ can be computed straightforwardly, and is found to be in good agreement with internal error from N-body simulations. For mildly nongaussian fields, we can expect that the use of a gaussian covariance matrix is still quite adequate, and in such cases a measurement of $\tau$ can reliably be made, subject to the requirement that the bias is linear on large scales. For highly non-gaussian fields, the gaussian hypothesis can be rejected, but the measurement of $\tau$ will be unreliable as the covariance matrix we use may neglect important terms. 
Application to data will probably have to wait for completion of the $2 \mathrm{dF}$ and Sloan surveys, as the distant-observer approximation is not a good one for the nearly all-sky PSCz survey. In addition, the high shot noise in the PSCz survey means that the expected errors on $\tau$ are large enough to make it hard to distinguish between GIC and some significantly non-gaussian fields. The $2 \mathrm{dF}$ and Sloan surveys should do much better. One could compute $\tau$ from volumes of side $100 h^{-1}$ Mpc with a random error of less than one per cent for a $\chi^{2}$ model as the one considered here, and could therefore put tight constraints on non-gaussian models. It would be helpful to apply this method to defect models, but unfortunately none is available at the required stage of evolution.

The major uncertainty in this analysis is the effect of bias. A non-linear bias term acts like a non-gaussian initial field, and, as we are using linear perturbation theory here, we cannot use polygons of different shape to lift the degeneracy, as done by MVH97 to lift the degeneracy between bias and gravitational evolution in the bispectrum. The best hope is to constrain a combination of initial non-gaussianity and quadratic bias, and to argue that if the measured value of $\tau$ is zero, then it would require a conspiracy unless both effects were absent. In principle it is possible to distinguish these two effects by considering differences at higher order in perturbation theory, as an Eulerian non-linear bias is applied to the evolved field, whereas a primordial non-gaussian field of the same mathematical form is applied to the initial field, and thus the evolution is different. It is an open question as to whether any realistic survey would have the signal-to-noise to do this.

\section{APPENDIX}

We consider the transformation of a gaussian field as follows:

$$
\delta(x) \longrightarrow \delta(x)+\epsilon \delta^{2}(x)
$$

This can be seen as the first two terms of a Taylor expansion of any non-gaussian field originating as a local mapping from a underlying gaussian one.

We choose a toy model for the power spectrum of the underlying gaussian field $P_{g}$ : a top hat function between some $k$ minimum and maximum. The power spectrum for the resulting field will then be:

$$
\begin{array}{r}
P(k)=P_{g}(k)+\frac{2}{(2 \pi)^{3}} \epsilon^{2} \int P_{g}\left(k^{\prime}\right) P_{g}\left(\left|\mathbf{k}-\mathbf{k}^{\prime}\right|\right) d^{3} k^{\prime} \sim \\
P_{g}(k)+2 \epsilon^{2} P_{g}(k)^{2} V_{k} /(2 \pi)^{3}
\end{array}
$$

where $V_{k}$ is the volume where the integrand in the previous equation is non-zero. The relevant quantities for our analysis, substituting the Dirac delta function by $V /(2 \pi)^{3}$ are the following:

$$
\begin{gathered}
\left\langle\delta_{\mathbf{k}_{1}} \delta_{\mathbf{k}_{2}} \delta_{\mathbf{k}_{3}} \delta_{\mathbf{k}_{4}}\right\rangle_{\text {conn. }} \sim\left(48 \epsilon^{2} P_{g}^{3}+\epsilon^{4} P_{g}^{4} V_{k} /(2 \pi)^{3}\right) V, \\
\text { SIGNAL } \equiv \tau \equiv \frac{\left\langle\delta_{\mathbf{k}_{1}} \delta_{\mathbf{k}_{2}} \delta_{\mathbf{k}_{3}} \delta_{\mathbf{k}_{4}}\right\rangle_{\text {conn. }} V}{\left\langle\delta_{\mathbf{k}_{1}} \delta_{\mathbf{k}_{2}}\right\rangle\left\langle\delta_{\mathbf{k}_{3}} \delta_{\mathbf{k}_{4}}\right\rangle} \sim
\end{gathered}
$$




$$
\begin{gathered}
\frac{48 \epsilon^{2} P_{g}^{3}+\epsilon^{4} P_{g}^{4} V_{k} /(2 \pi)^{3}}{\left(P_{g}^{2}+4 \epsilon^{2} P_{g}^{3} V_{k} /(2 \pi)^{3}+4 \epsilon^{4} P_{g}^{4}\left[V_{k} /\left(2 \pi^{3}\right)\right]^{2}\right)} \\
\text { NOISE } \simeq \sqrt{(2 \pi)^{3} V} \frac{2}{\sqrt{k_{\text {max }}^{3}-k_{\text {min }}^{3}}}
\end{gathered}
$$

The covariance matrix is dominated by the gaussian terms if $\left\langle\delta_{\mathbf{k}_{1}} \delta_{\mathbf{k}_{2}} \delta_{\mathbf{k}_{3}} \delta_{\mathbf{k}_{4}}\right\rangle_{\text {conn. }} \ll\left\langle\delta_{\mathbf{k}_{1}} \delta_{\mathbf{k}_{2}}\right\rangle\left\langle\delta_{\mathbf{k}_{3}} \delta_{\mathbf{k}_{4}}\right\rangle$, but at the same time we want to maximize the signal-to-noise ratio.

This considerations suggest that the best choice is: $P(k)=2(2 \pi)^{3}$ if $0.1<k<0.837$ and zero elsewhere and $\epsilon=0.4$. This allows us to use the gaussian covariance matrix, but also to have a reasonable signal-to-noise for the non-gaussian component from a single box of size of a few tens of Mpc.

\section{Shot Noise}

In the application of the method to a real survey the shot noise might be important. Here we report how to modify the relevant expressions in the presence of shot noise. In what follows $\bar{n}$ denotes the average number of particles per unit volume, and the superscript $d$ indicates that these results apply to discrete, point processes. We follow the generating functional approach of MVH97 to find the following:

$$
\begin{gathered}
\left\langle\delta_{i} \delta_{j}\right\rangle_{c}^{d} \longrightarrow(2 \pi)^{3}\left[P\left(k_{i}\right)+\frac{1}{\bar{n}}\right] \delta^{D}\left(\mathbf{k}_{i}+\mathbf{k}_{j}\right) \\
\left\langle\delta_{l} \delta_{m} \delta_{n}\right\rangle_{c}^{d} \longrightarrow(2 \pi)^{3} \delta^{D}\left(\mathbf{k}_{l}+\mathbf{k}_{m}+\mathbf{k}_{n}\right) \times \\
\left\{B_{l m n}+\frac{1}{\bar{n}}\left[P\left(k_{l}\right)+P\left(k_{m}\right)+P\left(k_{n}\right)\right]+\frac{1}{\bar{n}^{2}}\right\},
\end{gathered}
$$

where $B_{l m n}$ denotes the Bispectrum,

$$
\begin{aligned}
&\left\langle\delta_{o} \delta_{p} \delta_{q} \delta_{r}\right\rangle_{c}^{d} \longrightarrow(2 \pi)^{3} \delta^{D}\left(\mathbf{k}_{o}+\mathbf{k}_{p}+\mathbf{k}_{q}+\mathbf{k}_{r}\right) \times \\
&\left\{\frac{1}{\bar{n}}\left[B_{(o+p) q r}+\text { perm. }(6 \text { terms })\right]+\right. \\
& \frac{1}{\bar{n}^{2}}\left[\left(P_{o+p+q}+\text { cyc. }(4 \text { terms })\right)+\right. \\
&\left.P_{o+p}+P_{o+q}+P_{o+r}\right]+ \\
&\left.\frac{1}{\bar{n}^{3}}\right\} \\
&\left\langle\delta_{1} \ldots \delta_{5}\right\rangle_{c}^{d} \longrightarrow(2 \pi)^{3} \delta^{D}\left(\mathbf{k}_{1}+\mathbf{k}_{2}+\mathbf{k}_{3}+\mathbf{k}_{4}+\mathbf{k}_{5}\right) \times
\end{aligned}
$$




$$
\begin{aligned}
& \left\{\frac{1}{\bar{n}^{2}}\left[B_{(1+2)(3+4) 5}+\text { perm. }(15 \text { terms })\right]+\right. \\
& \left.\frac{1}{\bar{n}^{2}}\left[B_{(1+2+3) 45}+\text { perm. }(10 \text { terms })\right]\right\}+ \\
& \frac{1}{\bar{n}^{3}}\left[\left(P_{1+2}+\text { cyc. }(10 \text { terms })\right)+\right. \\
& \left.+P_{1}+P_{2}+P_{3}+P_{4}+P_{5}\right]+ \\
& \left.\frac{1}{\bar{n}^{4}}\right\}
\end{aligned}
$$

$$
\begin{aligned}
& \left\langle\delta_{1} \ldots \delta_{6}\right\rangle_{c}^{d} \longrightarrow(2 \pi)^{3} \delta^{D}\left(\mathbf{k}_{1}+\ldots+\mathbf{k}_{6}\right) \times \\
& \left\{\frac { 1 } { \overline { n } ^ { 3 } } \left[B_{12(3+\ldots+6)}+\text { perm. }(15 \text { terms })+\right.\right. \\
& B_{(1+2)(3+4)(5+6)}+\text { perm. (15 terms.) }+ \\
& B_{1(2+3)(4+5+6)}+\text { perm. (60 terms)] }+ \\
& \frac{1}{\bar{n}^{4}}\left[P_{1}+\ldots+P_{6}+\right. \\
& P_{1+2}+\text { perm. (15 terms) }+ \\
& \left.P_{1+2+3}+\text { perm. }(10 \text { terms })\right]+ \\
& \left.\frac{1}{\bar{n}^{5}}\right\} \text {. } \\
& \left\langle\delta_{1} \ldots \delta_{8}\right\rangle_{c}^{d} \longrightarrow(2 \pi)^{3} \delta^{D}\left(\mathbf{k}_{1}+\ldots+\mathbf{k}_{8}\right) \times \\
& \left\{\frac { 1 } { \overline { n } ^ { 5 } } \left[B_{12(3+\ldots+8)}+\text { perm. }(28 \text { terms })+\right.\right. \\
& B_{1(2+3)(4+\ldots+8)}+\text { perm. }(168 \text { terms })+ \\
& B_{1(2+3+4)(5+\ldots+8)}+\text { perm. }(280 \text { terms })+ \\
& B_{(1+2)(3+4+5)(6+7+8)}+\text { perm. }(280 \text { terms })+ \\
& \left.B_{(1+2)(3+4+5+6)(7+8)}+\text { perm. }(210 \text { terms })\right]+ \\
& \frac{1}{\bar{n}^{6}}\left[P_{1}+\ldots+P_{8}+\right. \\
& P_{1+2}+\text { perm. (28 terms) }+ \\
& P_{1+2+3}+\text { perm. (56 terms) }+ \\
& P_{1+2+3+4}+\text { perm. }(35 \text { terms })+ \\
& \left.\frac{1}{\bar{n}^{7}}\right\} \text {. }
\end{aligned}
$$

Neglecting shot noise when it is not negligible has two effects. The main effect is to overestimate $H$. E.g., the generalization of (8) is $T \rightarrow T_{S N}$, where

$$
(2 \pi)^{3} T_{S N}=
$$




$$
\begin{aligned}
& (2 \pi)^{3}\left(P\left(k_{1}\right)+\frac{1}{\bar{n}}\right)\left(P\left(k_{3}\right)+\frac{1}{\bar{n}}\right) \delta^{D}\left(\mathbf{k}_{1}+\mathbf{k}_{2}\right) \delta^{D}\left(\mathbf{k}_{3}+\mathbf{k}_{4}\right) \\
& +\left\langle\delta_{1} \ldots \delta_{4}\right\rangle_{c}^{d}
\end{aligned}
$$

The second effect is that the errors are underestimated. In particular, even in the gaussian case, there are additional connected terms in the correlations arising from shot noise.

\section{Selection function}

The above analysis is valid for volume-limited samples, where the mean number density of galaxies is independent of position. In a realistic catalogue the presence of a varying selection function makes the mean density position-dependent and might even induce spurious detection of non-gaussianity. However it is possible to treat this effect accurately by the generating functional approach as in MVH97. If $n(\mathbf{x})$ is the mean density of such a catalogue, following Feldman et al. (1994), we can define a working fluctuation field composed by subtracting from the real catalogue a synthetic catalogue with no clustering, but with the same selection function, and then weighting suitably the combination:

$$
F(\mathbf{x})=\gamma w(\mathbf{x})\left[n(\mathbf{x})-\alpha n_{s}(\mathbf{x})\right]
$$

where $w(\mathbf{x})$ is an arbitrary weighting function, $\gamma$ is a normalization factor, $n_{s}(\mathbf{x})=n(\mathbf{x}) / \alpha$, and $\alpha$ is the "dilution" of the synthetic catalogue. We will then consider the limit $\alpha \longrightarrow 0$ to avoid shot noise in the synthetic catalogue. The $n$-point correlation functions in Fourier space are easily calculated by considering $F$ as the superposition of the process $f=\gamma w(\mathbf{x}) n(\mathbf{x})$ and $f_{s}=-\alpha \gamma w(\mathbf{x}) n_{s}(\mathbf{x})$. The generating functional for $F$ will then be: $\mathcal{Z}_{F}(\mathcal{J})=\mathcal{Z}_{f}(\mathcal{J}) \mathcal{Z}_{f_{s}}(-\alpha \mathcal{J})$. Ignoring shot noise,

$$
\mathcal{Z}_{f_{s}}(-\alpha \mathcal{J})=-\alpha i \int d^{3} x \mathcal{J}_{s}
$$

where $\mathcal{J}_{s}=\gamma w(\mathbf{x}) n_{s}(\mathbf{x}) \sum_{m} s_{m} \exp \left(-i \mathbf{k}_{m} \cdot \mathbf{x}\right)$, and the ansatz for the generating functional for the field $f$ is:

$$
\begin{gathered}
\mathcal{Z}_{f}[\mathcal{J}]= \\
\exp \left[i \int d^{3} x \mathcal{J}(\vec{x})-\frac{1}{2} \int d^{3} x d^{3} x^{\prime} \mathcal{J}(\vec{x}) \mathcal{J}\left(\vec{x}^{\prime}\right) \xi_{\text {conn. }}^{(2)}\left(\vec{x}, \vec{x}^{\prime}\right)\right. \\
-\frac{i}{6} \int d^{3} x d^{3} x^{\prime} d^{3} x^{\prime \prime} \mathcal{J}(\vec{x}) \mathcal{J}\left(\vec{x}^{\prime}\right) \mathcal{J}\left(\vec{x}^{\prime \prime}\right) \xi_{\text {conn. }}^{(3)}\left(\vec{x}, \vec{x}^{\prime}, \vec{x}^{\prime \prime}\right) \\
\left.+\frac{1}{24} \int d^{3} x d^{3} x^{\prime} d^{3} x^{\prime \prime} d^{3} x^{2} \mathcal{J}(\vec{x}) \mathcal{J}\left(\vec{x}^{\prime}\right) \mathcal{J}\left(\vec{x}^{\prime \prime}\right) \cdots \mathcal{J}\left(\vec{x}^{l}\right) \xi_{\text {conn. }}^{(4)}\left(\vec{x}, \cdots, \vec{x}^{2}\right)\right]
\end{gathered}
$$

Following the same procedure as in Matarrese et al. (1997) it is possible to obtain the $n$-point function in Fourier space by differentiating the generating functional; shot noise can be easily included by modifying the generating functional .

The $\tau$ in presence of a spatially-varying selection function therefore becomes:

$$
\tau \longrightarrow \tau \frac{I_{44}}{I_{22}^{2}}
$$


where

$$
I_{i j} \equiv \int d^{3} \mathbf{x} w^{i}(\mathbf{x}) n^{j}(\mathbf{x})
$$

In general the effect of a spatially-varying selection function can be summarized as follows:

$$
\begin{aligned}
\gamma^{2} & =1 / I_{22} \\
V & \longrightarrow \frac{I_{N N}}{I_{22}^{N / 2}} ; \delta^{D} \longrightarrow \frac{I_{N N}}{(2 \pi)^{3} I_{22}^{N / 2}} \\
\frac{1}{\bar{n}^{q}} & \longrightarrow \frac{I_{N(N-q)}}{I_{N N}}
\end{aligned}
$$

\section{Acknowledgments}

LV acknowledges NASA grant NAG5-7154 and TMR fellowship. We are grateful to Sabino

Matarrese, Avery Meiksin, M. Strauss and the anonymous referee for helpful comments. The simulation with gaussian initial conditions was obtained from the data bank of cosmological Nbody simulations provided by the Hydra consortium (http://coho.astro.uwo.ca/pub/data.html) and produced using the Hydra N-body code (Couchman et al. 1995). LV thanks Alison Stirling for providing the N-body simulation with non-gaussian initial conditions.

\section{REFERENCES}

Albrecht, A. \& Stebbins, A. 1992, Phys. Rev. Lett., 68, 2121

Allen, T. J., Grinstein, B., \& Wise, M. B. 1987, Phys. Lett. B, 197, 66

Amendola, L. 1996, MNRAS, 283, 983

Avelino, P. P. 1997, ApJ, 487, 18

Banday, A. J., Zarubi, S., \& Gorski, K. M. 1999, astro-ph, 990870, preprint

Bernardeau, F. 1994, ApJ, 433, 1

1992, In new insight into the Universe, ed. Martinez, Portilla Saez, Springer-Verlag

Bouchet, F., Juszkiewicz, R., Colombi, S., \& Pellat, R. 1992, ApJ, 394, L5

Brillinger, D. R. 1975, Time series:Data Analysis and Theory (Holt, Rienehart and Winston, New York) 
Brillinger, D. R. \& Rosenblatt, M. 1967, in Advanced Seminar on Spectral Analysis of Time Series, ed. B. Harris (Wiley, New York), 153-232

Catelan, P., Lucchin, F., Matarrese, S., \& Moscardini, L. 1995, MNRAS, 276, 39

Catelan, P. \& Moscardini, L. 1994, ApJ, 436, 5

Catelan, P. \& Sherrer, J. R. 1995, ApJ, 445, 1

Chiu, W. A., Ostriker J. P. \& Strauss, M. A. 1998, ApJ, 494, 479

Chodorowski, M. J. \& Bouchet, F. R. 1996, MNRAS, 279, 563

Coles, P., Moscardini, L., Plionis, M., Lucchin, F., Matarrese, S., \& Messina, A. 1993, MNRAS, 260,572

Colless, M. 1996, in Wide Field Spectroscopy, ed. M. Kontizas \& E. Kontizas (Kluwer), 227

Couchman, H. M. P., Thomas, P. A., \& Pearce, F. R. 1995, ApJ, 452, 797

Falk, T., Rangarajan, R., \& Srednicki, M. 1993, ApJ(Lett), 403, 1

Fan, Z. \& Bardeen, J. M. 1994, American Astronomical Society Meeting, 184, 62.06

Feldman, H. A., Kaiser, N., \& Peacock, J. A. 1994, ApJ, 426, 23

Ferreira, P., Magueijo, J., \& Gorski, K. 1998, ApJLett, 503, 1

Fry, J. N. 1984, ApJ, 279, 499

Fry, J. N. \& Scherrer, R. J. 1994, ApJ, 429, 36

Gangui, A., Lucchin, F., Matarrese, S., \& Mollerach, S. 1994, ApJ, 430, 447

Gaztanaga, E. \& Fossalba, P. 1998(a), MNRAS, 301, 503

-. 1998(b), MNRAS, 301, 524

Goroff, M. H., Grindstein, B., Rey, S. J., \& Wise, B. 1986, ApJ, 311, 6

Hamilton, A. J. S. 1992, ApJ(Lett), 385, L5

Hamilton, A. J. S. \& Tegmark, M. 2000, preprint, astro-ph, 0008392

Hatton, S. J. \& Cole, S. 1998, MNRAS, 296, 10

Heavens, A. F. 1998, MNRAS, 299, 805

Heavens, A. F. \& Taylor, A. N. 1995, MNRAS, 275, 483 
Hill, C. T., Schramm, D. N., \& Fry, J. N. 1989, Comments Nucl. Part. Phys., 19, 25

Hinshaw, G., Kogut, A., Gorski, K., Banday, A., Bennett, C., Lineweaver, C., Lubin, P., Smoot, G., \& Wright, E. 1994, ApJ, 431, 1

Kim, R. \& Strauss, M. A. 1998, ApJ, 493, 39

Kaiser, N. 1987, MNRAS, 227, 1

Kofman, L. \& Pogosyan, D. Y. 1988, Phy. Rev. Lett. B, 214, 508

Kogut, A., Banday, A., Bennett, C., Gorski, K., Hinshaw, G., Smoot, G., \& Wright, E. 1996, ApJ, 464, L29

Lii, K. S., Rosenblatt, M., \& Atta, C. V. 1976, J. Fluid Mech., 77, 45

Lokas, E. L., Juszkiewicz, R., Weinberg, D. H., \& Bouchet, F. R. 1995, MNRAS, 274, 744

Luo, X. 1994, Phys. Rev., D49, 3810

Luo, X. \& Schramm, D. N. 1993, ApJ, 408, 33

Matarrese, S., Lucchin, F., Messina, A., \& Moscardini, L. 1991, MNRAS, 253, 35

Matarrese, S., Verde, L., \& Heavens, A. 1997, MNRAS, 290, 651

Matarrese, S., Verde, L., \& Jimenez, R. 2000, ApJ, 541, 10

Moscardini, L., Matarrese, S., Lucchin, F., \& Messina, A. 1991, MNRAS, 248, 424

Moscardini, L., Messina, A., Londrillo, P., Lucchin, F., \& Matarrese, S. 1990, MNRAS, 245, 244

Peacock, J. A. \& Dodds, S. J. 1994, MNRAS, 267, 1020

Peebles, P. J. E. 1999a, ApJ, 510, 523

-. 1999b, ApJ, 510, 531

Press, W. H., Teukolosky, S. A., Vetterling, W. T., \& Flannery, B. P. 1992, Numerical recipes in Fortran, 2nd edition (C.U.P., Cambridge)

Robinson, J., Gawiser, E., Silk, J. 2000, ApJ, 532, 1

Salopek, D., Bond, J. R., \& Bardeen, J. M. 1989, Phys. Rev. D, 40, 1753

Saunders, W., Sutherland, W., Efstathiou, G., Tadros, H., Maddox, S., McMahon, R., White, S., Oliver, S., Keeble, O., Rowan-Robinson, M., \& Frenk, C. 1998, MNRAS, in preparation

Scherrer, R. J. 1992, ApJ, 390, 330 
Scherrer, R. J. \& Shaffer, R. K. 1995, ApJ, 446, 44

Scoccimarro, R., Colombi, S., Fry, J. N., Frieman, J. A., Hivon, E., \& Melott, A. 1998, ApJ, 496, 586

Scoccimarro, R., Couchman, H. M. P., \& Frieman, J. A. 1999, ApJ, 517, 531

Smoot, G. F., Tenorio, L., Banday, A., Kogut, A., Wright, E. L., Hinshaw, G., \& Bennett, C. L. 1994, ApJ, 437, 1

Stirling, A. J. \& Peacock, J. A. 1996, MNRAS, 283, 99

Taruya, A., Magira, H., Jing, Y. P., Suto, Y. 2000, astroph-0012511

Tegmark, M. \& Bromley, B. C. 1999, ApJ(Lett), 518, 69

Turok, N. 1989, Phys. Rev. Lett., 63, 2625

Vachaspati, T. 1986, Phys. Rev. Lett., 57, 1655

Verde, L., Heavens, A., Matarrese, S., \& Moscardini, L. 1998, MNRAS, 300, 747

Verde, L., Jimenez, R., Kamionkowski, M., \& Matarrese, S. 2000a, to be submitted to MNRAS, astro-ph/0011180

Verde, L., Kamionkowski, M., Mohr, J. J., \& Benson, A. J. 2000b, MNRAS in press, astro$\mathrm{ph} / 0007426$

Verde, L., Wang, L., Heavens, A. F., \& Kamionkowski, M. 2000c, MNRAS, 313, 141

Vilenkin, A. 1985, Phys. Rep., 121, 263

Vogeley, M. S., Park, C., Geller, M. J., Huchra, J. P., \& Gott III, J. R. 1994, ApJ, 420, 525

Weinberg, D. H. \& Cole, S. 1992, MNRAS, 259, 652

Willick, J. A. 2000, ApJ, 530, 80

York et al., astro-ph/0006396 
Fig. 1.-The 4-point correlation function in Fourier space (disconnected and connected) $\left\langle D_{\alpha}\right\rangle \equiv\left\langle\delta_{\mathbf{k}_{1}} \ldots \delta_{\mathbf{k}_{4}}\right\rangle$ is approximated by averaging square configurations with binned wavevectors from a CDM-like N-body simulation (solid line). Also shown is the linear perturbation theory prediction for gaussian initial conditions i.e. $\mu_{\alpha}(\tau=0)$ from equation $(9)$, for a square configuration of wavevectors. Errors are errors in the mean for each bin. For this square configuration, linear perturbation theory breaks down around $k=0.67 h \mathrm{Mpc}^{-1}$.

Fig. 2.-Minimum $\chi^{2}$ analysis for the parameter $\tau$ for the gaussian CDM-like N-body simulation. Only the 'square' configuration for the trispectrum has been considered here and $0.15 \leq$ $k \leq 0.67$ (in units of $\mathrm{h} \mathrm{Mpc}^{-1}$ ). $\tau$ is a measure of the connected part of the trispectrum; for gaussian initial conditions $\tau=0$ (vertical line). To produce this graph, we use the gaussian initial conditions covariance matrix to compute the likelihood (equation 11). See sections 2.1 and 3 for further details.

Fig. 3.-The $\chi^{2}$ analysis for 460 volumes of $20 h^{-1}$ Mpc side (dot-dashed line) and 266 volumes of $24 h^{-1} \mathrm{Mpc}$ side (dashed line) for $0.1<k<0.837$ (in units of $\mathrm{h} \mathrm{Mpc}^{-1}$ ). The $y$ axis is normalized so that $1-\sigma$ limits are at $y=1$. The predicted value for $\tau$ is $\tau \simeq 1040$ (vertical lines). This illustrates the scaling with the volume of the error on $\tau$ (see Section 3.1 for more details).

Fig. 4. - Minimum $\chi^{2}$ analysis for the parameter $\tau$ from the redshift-space unbiased CDM-like N-body simulation (see text for details). Only the square configuration has been considered here. The value for the velocity dispersion parameter $\sigma \simeq 980 \mathrm{~km} / \mathrm{s}$ although it is slightly scale-dependent. The range of $k$-vectors considered is $k \leq 0.67 h \mathrm{Mpc}^{-1}$.

Fig. 5.- Quadrupole-to-monopole ratio for the trispectrum $R_{T}$ for degenerate square configuration of a GIC simulation. The continuous line is the theoretical $R_{T}$ using the redshift-space distortions as in VHMM98 (see section 4.1 for details). 


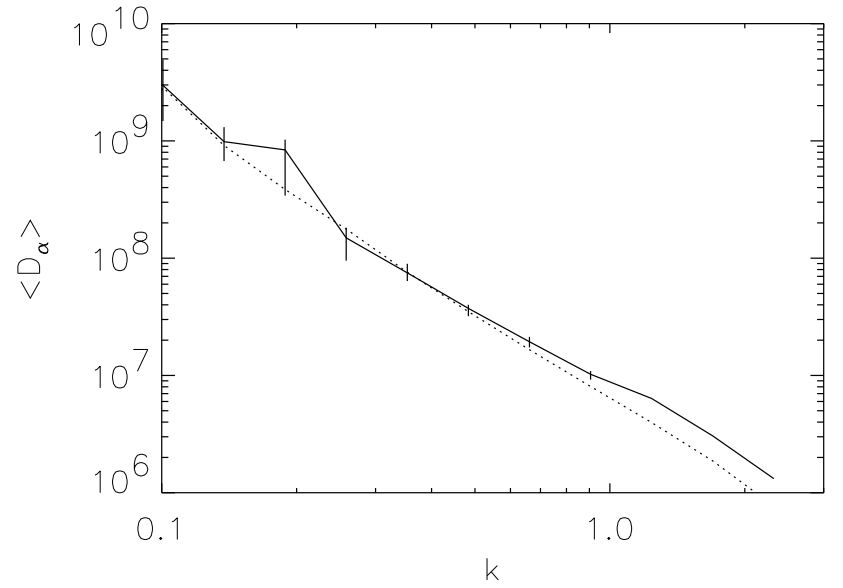

Fig. 1.- 


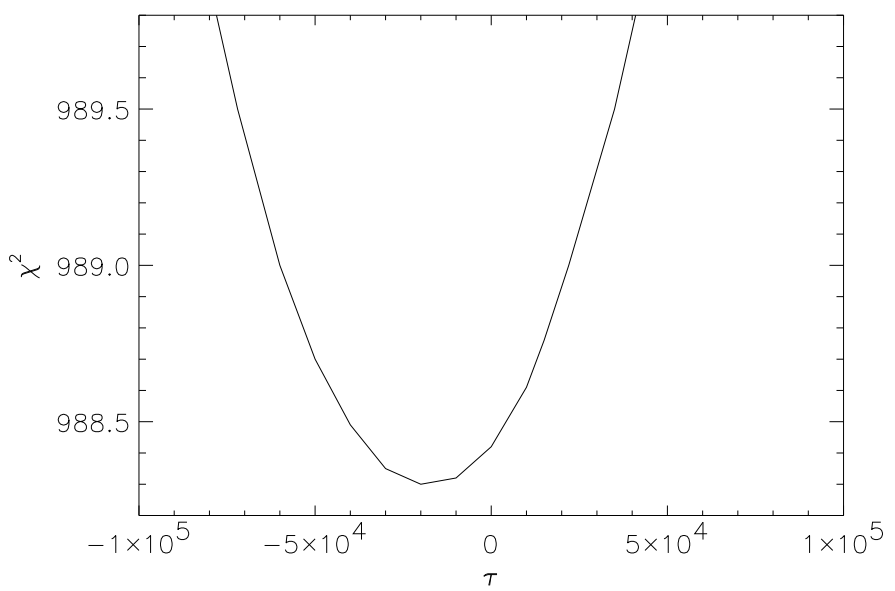

Fig. 2.- 


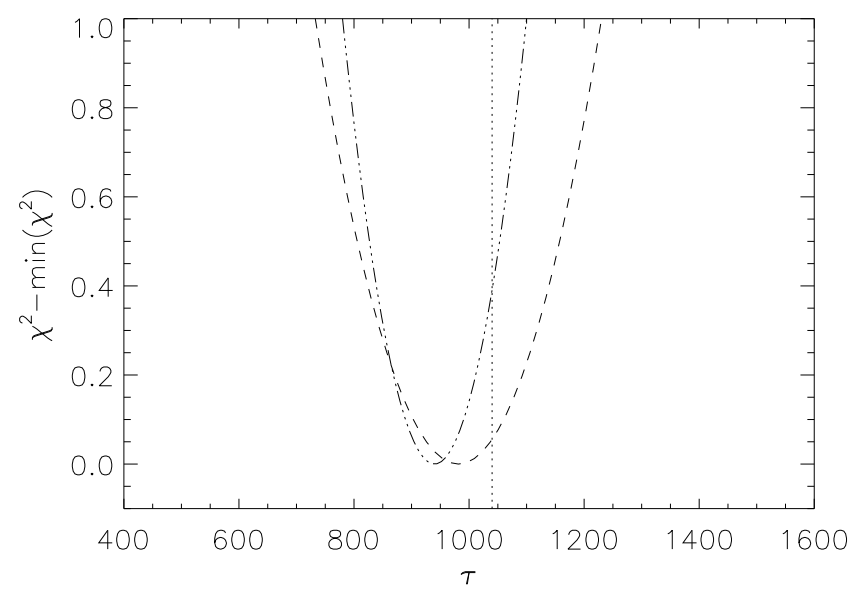

Fig. 3.- 


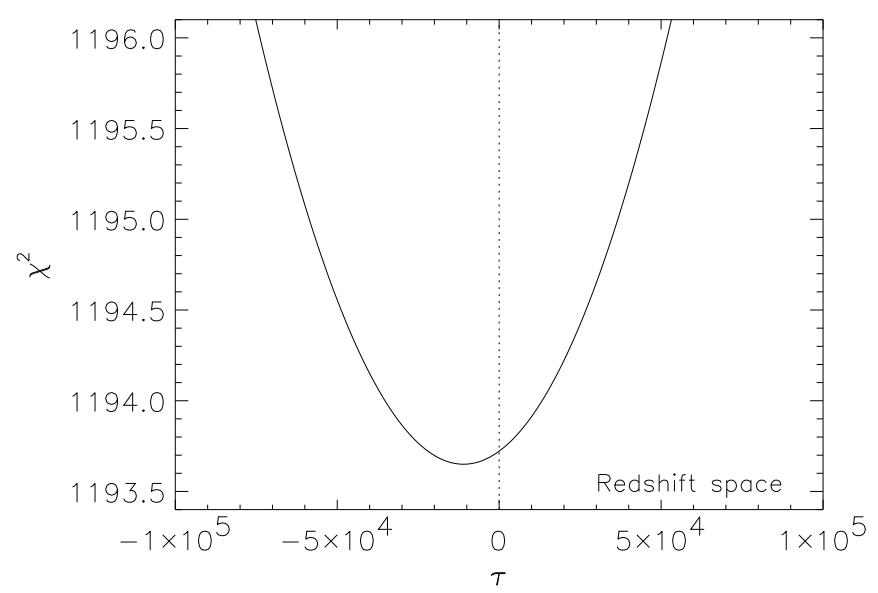

Fig. 4.- 


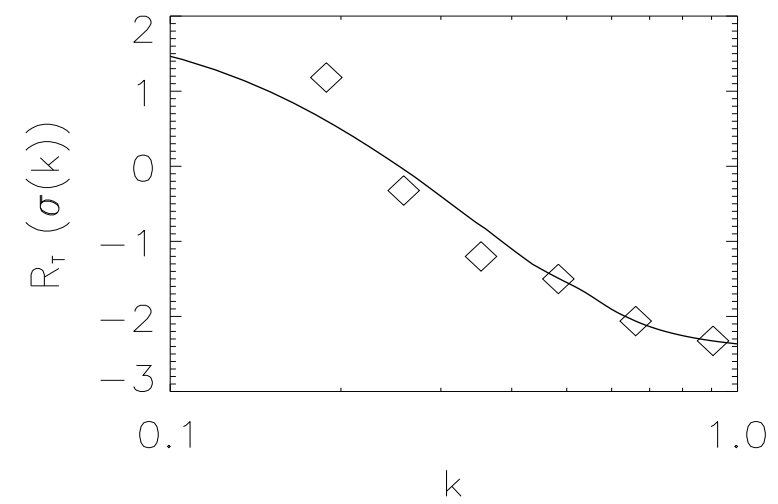

Fig. 5.- 